\title{
Next generation polymeric flocculants for thickening and dewatering
}

H. Kolla Kemira, USA

A. Mahmoudkhani Kemira, USA

P. Watson Kemira, USA

M. Awad Kemira, USA

P. O'Neill Kemira, Canada

L. Moore Kemira, USA

\begin{abstract}
The management of tailings streams has taken an increasingly important role in the minerals industry in recent years. The global issue of water scarcity and tightening of regulations governing the disposal of waste waters has significantly contributed to a focus on the development of thickener technologies that is not only widely accepted but also economically advantageous. The operational desire for high density paste thickeners involves dewatering (water re-use), underflow density (pipeline transport), and stacking (deposition processes), all of which are governed by rheology. Although thickener technology has proved to be effective, in many cases, it is not efficient by itself.

The addition of chemical agents known as rheology modifiers or flocculants has shown to be instrumental in improving the overall performance efficiency of the thickening process. These flocculants are generally high molecular weight water soluble polymers that adsorb onto particle surfaces and bridge them together to form large aggregates, thus facilitating flocculation. Most of the commercially available flocculants are generically designed to perform across a broad range of mineral solids (mineralogy), but are not capable of targeting multiple performance criteria. However, a range of next-generation flocculants has been developed; these excel at multiple performance criteria for a particular mineralogy. This paper discusses the dewatering performance, stacking capability, and changes in the underflow rheology of processed gold tailings when treated with various flocculants (traditional versus next-generation).
\end{abstract}

\section{Introduction}

Mineral thickening and clarification is usually achieved by thickeners that operate on the principle of gravity sedimentation. The most common use of this technology is employed in tailing streams to achieve surface stacking and water recovery (dewatering), both of which have become very important economic criteria in the mining industry today (Mensah-Addai and Ralston, 2006). Dewatering has become a topic of much focus around the globe, as water stresses can limit the quality and quantity of available water. Additionally, transporting fresh water over large distances is not always feasible or economical. In order to address this, the thickening process produces an overflow that can be recycled back into the main stream and a thickened underflow that can be pumped into a waste pond. The thickened slurry's high solids concentration increases the material's yield stress, which aids mechanical properties at the deposition site, but can also negatively affect the pumpability of the underflow solids.

Although over the past several years, tremendous advancements in the engineering aspects of the thickeners have improved their operational performance, it is the introduction of chemical aids known as rheology modifying agents, or thickening agents, that has significantly improved the overall efficiency of this process (Berger et al., 2011). Commonly used rheology modifiers (also known as flocculants) are very high molecular weight water soluble polymers. These polymeric flocculants improve the thickener 
efficiency by increasing the rate of initial compaction of the slurry particles through the formation of an aggregate network derived from polymer-slurry interaction. These aggregated particles settle faster than the non-flocculated particles because of their higher mass-to-surface area ratio (Berger et al., 2011). This mechanism facilitates the formation of a highly structured and compacted zone with a volume fraction much lower than that of the non-flocculated slurry. These compacted zones promote improved dewatering due to the formation of channels between the flocs, which facilitate upward percolation (Berger et al., 2011). Due to these and many other advantages, the use of polymeric flocculants has become a common practice and, more recently, a prerequisite in the mining industry; its benefits are well documented (Pearse, 2003; Pearse and Barnett, 1980).

Although the use of flocculants improves settling and dewatering rates, it also significantly increases the yield stress of the settled solids compared to that of untreated solids. Such changes may adversely impact the performance of the rake, underflow pump, and pipeline transport, impacting the discharge of treated material (Schoenbrunn, 2011). Therefore, it is important to design and formulate flocculants that are capable of balancing flow, compaction, and dewatering without impacting the underflow rheology characteristics.

The focus of this study is to address dewatering and stacking characteristics using flocculants specifically designed for the mineralogy of the tailings used. In this paper, we demonstrate how rheology measurements, particle size distribution studies, dewatering measurements, and stackability tests provide the feedback necessary to design and develop flocculants that produce the requisite settling rates, dewatering capability, and stackability, while maintaining an underflow rheology (underflow density of $\sim 55-60 \%$ solids) that does not negatively affect the pumping and transportation of minerals.

\section{$2 \quad$ Materials and methods}

\section{$2.1 \quad$ Settling tests}

Tailings and water for this study were obtained from a gold processing mill in Canada. Settling tests were performed in a $2 \mathrm{~L}$ graduated cylinder. Tailings were first diluted (using the mill water) to $\sim 20-25 \%$ solids and homogenised (by plunging) prior to flocculant addition. The exact solids concentration was also calculated and the right amount of flocculant weighed prior to flocculant addition. The flocculant was then added quickly ( 5 seconds) and plunging was continued for 30 seconds thereafter, with the mixing time dictated by the gold processing mill. The flocculation process started and the cylinder was set aside undisturbed until the end of the experiment ( 3 hours). No rakes were used during the test. The bed volume of the settling tailings was measured at 10,30,60, and 180 minutes. The bed volume at the end of 3 hours was used to calculate the tailings concentration in the underflow (\% solids). Turbidity and suspended solids in the supernatant were also measured at the end of 3 hours. The supernatant was decanted and stored. The settled tailings were carefully poured into a steel tray to test for stackability. After measuring the base diameter of the stacked tailings (where applicable), samples from this were extracted for analysis of further characteristics, such as oven solids, capillary suction time (CST), particle size distribution (PSD), rheometry, and compression.

\subsection{Dynamic light scattering}

Aggregation of fine tailings was investigated using an inline particle size measurement by dynamic light scattering. Particle size distributions (PSD) were determined with a Beckman Coulter model LS-230, which measures the angular dependence of scattered light (mainly in the forward direction). A fine fraction of tailing solids was initially separated from a coarser fraction by gravitation over a period of 4 hours. Then a $1.0 \mathrm{~mL}$ sample was dispersed in the container flow loop of the analyser containing tap water (approximately $700 \mathrm{~mL}$ ) without additional chemical dispersants. Particle size distributions were computed as equivalent-sphere size distributions based on Mie scattering and Fraunhofer diffraction formalisms applied to the scattering data (Malvern Instrument Handbook, 1997). Measurements were taken during each test at 30,60, 90, and 120 seconds after addition of the solids to the diluent under continuous 
agitation at a single flow speed. This procedure allowed us to assess whether a steady-state size distribution had been achieved. In most cases, particle size distributions reached a steady state after 90 seconds of mixing and this was used for comparisons. Then $0.2 \mathrm{~mL}$ of polymer flocculant solution $(0.4 \%$ in water) was added to the above diluted slurry, and particle size distribution was re-measured. Offline particle size measurement was done using flocculated solids obtained from treatment of tailings in $1 \mathrm{~L}$ graduated cylinders after 12 hours settling.

\subsection{Capillary suction time}

The dewatering properties of materials excised from settled beds of treated tailings were measured using a capillary suction time (CST) method as described in Standard Method APHA 2710 G (American Public Health Association, 1999). CST measurements were carried out using a CST instrument from Venture Innovations Inc., California, USA. The CST is the time interval it takes an aqueous solution to traverse between two radial positions in a filter paper (Whatman No. 40, $9 \mathrm{~cm}$ ) under the influence of capillary suction (Scholz, 2005). A low CST value implies good sludge dewatering, i.e., the water from the paste releases quickly with little impediment. Each measurement was conducted at least in triplicate, and the average was presented.

\subsection{Stackability index using squeeze/compression test}

The squeeze test is a simple compression test that is often carried out on cylindrical or cubic samples. The apparatus consists of two coaxial parallel plates, without any rotation. The upper disc can be displaced at controlled constant velocity, while the lower one remains stationary. The squeezing of the sample between the two plates induces a radial and axial flow. $D$ is the diameter of the sample cube, $H$ is the height of the sample, $F$ is the compression load applied on the plates, and $c$ is the compression speed (see Figure 1 ).

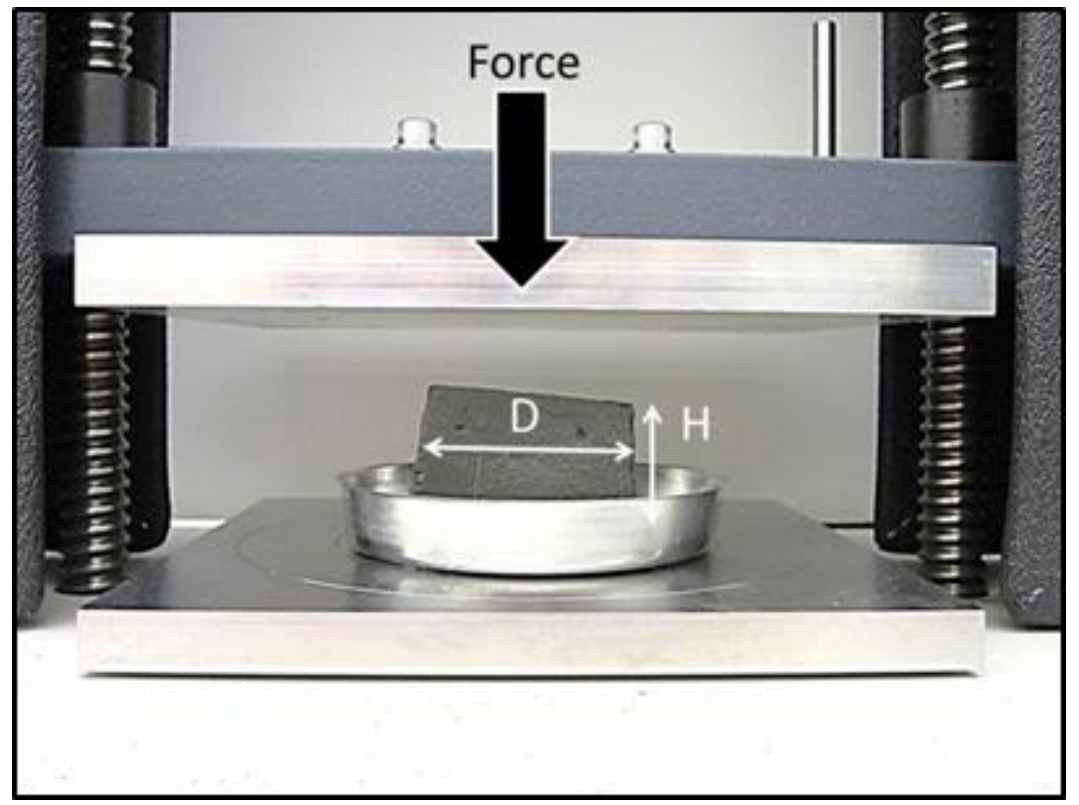

Figure 1 Squeeze/compression test geometry

Flocculated tailing paste samples were transferred into $50 \mathrm{~mm}$ plastic cube moulds, covered, and left for 24 hours for curing. Released water was decanted and the paste cube was gently removed and taken for measurement. Sample height, in most cases, was $40-45 \mathrm{~mm}$. A Compression Tester instrument model 17-70 from Testing Machine Inc. (New York, USA) was used at peak height mode. The peak height test deforms a specimen by a preset amount to achieve a deformation of $5 \mathrm{~mm}$ and reports the highest force required to realise that deformation. The compression speed (c) was set at $12.7 \mathrm{~mm} / \mathrm{min}$. Each compression measurement was conducted at least in triplicate, and the average was presented. 


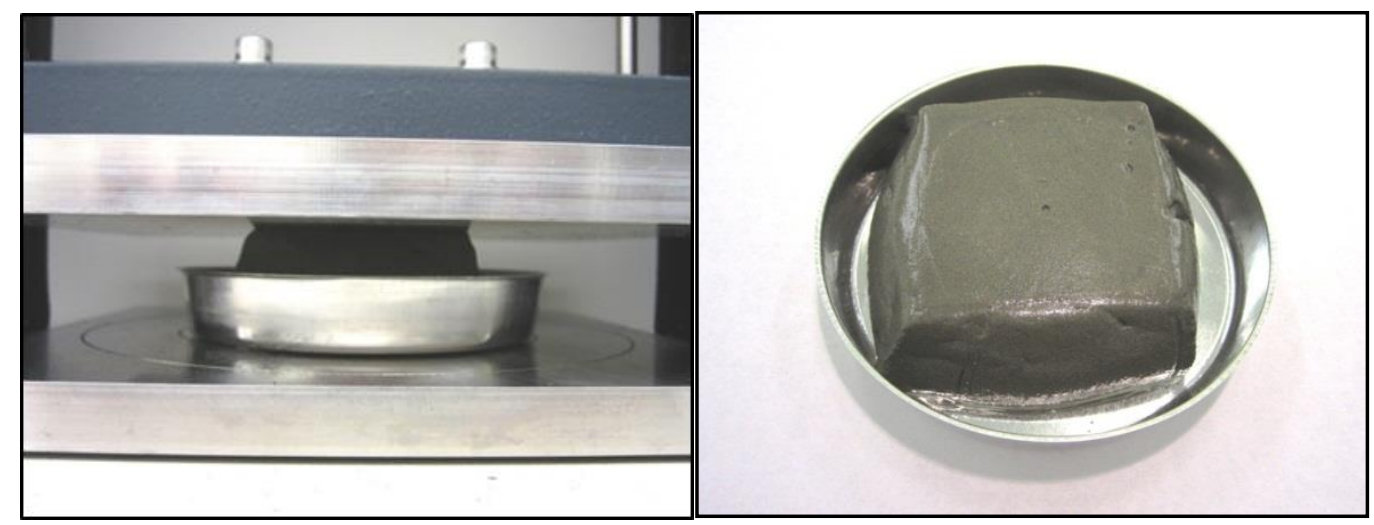

Figure 2 Compression and deformation of paste cube

\subsection{Dynamic rheometry}

An Anton-Paar MCR 300 rheometer equipped with a six-bladed Anton Paar ST 22-6V-16/106 spindle (equivalent to the Thermo Haake FL 100 spindle) was used to measure the yield stress, thixotropic recovery, and viscosity profiles of thickened tailings.

For the yield stress measurements, a $50 \mathrm{~mL}$ centrifuge tube containing the sample was decanted of free water and then clamped inside the rheometer's sample holder. Next, the spindle was lowered into the middle of the sample, stopping at $5 \mathrm{~cm}$ from the bottom of the tube. The rotational shear stress was steadily increased, and the amount of stress needed to result in a non-linear strain response was defined as the yield stress. For thixotropic recovery tests, the sample was left in the rheometer for either 15 or 30 minutes, and the yield stress measurement was re-run.

Thixotropic recovery was calculated by dividing the yield stress at 15 or 30 minutes by the initial yield stress. Viscosity profiles were measured after samples had undergone yield stress testing. The paste viscosity was measured as the sample was sheared at rates from 1 to $500 \mathrm{~s}^{-1}$. Yield stresses and viscosities were normalised by the solids concentration of the pastes to facilitate sample-to-sample comparisons.

\section{$3 \quad$ Results and discussion}

Clay mineralogy and morphology can have a dramatic effect on how polymers flocculate and modify the rheology of mineral slurries. Particle size distribution, surface charge, particulate shape, and chemical composition are some of the factors that are important to consider while designing flocculants and/or trying to modify the rheology of the tailings slurry (Sofra and Boger, 2011). Therefore, it is important that the flocculant design focus on targeting multiple performance criteria tailored to specific mineralogy. Conventional flocculants are known to increase the settling rate in a thickener to acceptable levels by the mechanism of binding fine particles together to form large aggregates, facilitating faster settling. However, a majority of these can cause water to be entrapped (within the floc and/or in the flocculated particle network) so that it is not fully expelled into the thickener bed, thus decreasing the dewatering capability. Additionally, the yield stresses of the networked structure formed in the bed can reach unacceptably high levels (Hogg, 1999). In the following paragraphs, we discuss the performance differences that the various polymers (including the incumbent, Polymer A) show on settling, dewatering, turbidity (water quality of the overflow), stacking, and underflow rheology (for transport and stackability).

\subsection{Settling characteristics}

Settling characteristics were determined using the procedure described in the materials and methods section. For this study, we have considered 9 polymer flocculants, $A, B, C, D, E, F, G, H$, and J. Polymer $A$ is the control incumbent product in the mill, flocculants $B, G, H$, and $J$ are acrylamide-based dry polymers, while $C, D, E$ and $F$ are acrylamide-based emulsion polymers. All polymers have been chosen to provide a representative cross-section of polymeric physical state (dry versus emulsion), charge, and molecular 
weight. In case of emulsion polymers, the dose was adjusted to compensate for active content. Among the 8 polymers (excluding $A$ ), B and $C$ have been synthetically modified in terms of both backbone chemistry and process chemistry to suit the mineralogy of the gold tailings used in this study. These polymers are the result of a lengthy evaluation of chemistry changes applied to the acrylamide based polymers. Mineral slurries usually contain significant amounts of fines that complicate the flocculation process, and effective flocculants interact with the fine and coarse particles. Table 1 shows the effect of flocculation with respect to underflow concentration (\% solids), turbidity, and solids concentration in the supernatant (\%).

\section{Table 1 Compaction and turbidity values for all flocculants}

\begin{tabular}{cccc}
\hline Sample ID & $\begin{array}{c}\text { Underflow solids } \\
\text { \% (at 3 hours) }\end{array}$ & $\begin{array}{c}\text { Turbidity - NTU } \\
\text { (Nephelometric } \\
\text { Turbidity Unit), } \\
\text { at 3 hours) }\end{array}$ & $\begin{array}{c}\text { \% Solids in the } \\
\text { overflow }\end{array}$ \\
\hline A & 69.67 & Out of range & 0.44 \\
B & 65.19 & 107 & 0.16 \\
C & 71.92 & 897 & 0.24 \\
D & 64.39 & 163 & 0.09 \\
E & 65.32 & 364 & 0.13 \\
F & ND & N/A & 0.83 \\
G & ND & N/A & N/A \\
H & 67.65 & Out of range & 0.61 \\
J & ND & N/A & N/A \\
\hline
\end{tabular}

Among all flocculants, $\mathrm{C}$ showed the best underflow concentration, resulting in a flocculated bed containing approximately $72 \mathrm{wt} \%$ solids, followed by $A$ at approximately $70 \mathrm{wt} \%$ solids. All other flocculants showed underflow concentration ranging from approximately 64 to $68 \mathrm{wt} \%$ solids. Flocculant B showed the best overflow clarity at $\sim 107$ NTU, followed by D at 160 NTU.

For Polymers $\mathrm{A}$ and $\mathrm{H}$, the supernatant contained such a large number of suspended particles that the turbidity was too high to measure, while Polymers F, G, and J showed extremely poor flocculation, so that no further measurements could be obtained. Therefore, no further tests were performed on these, and for all further comparisons, only Polymers $\mathrm{A}, \mathrm{B}, \mathrm{C}, \mathrm{D}, \mathrm{E}$, and $\mathrm{H}$ are reported.

The settling rate is important in order to determine the time required for an acceptable solid-liquid separation. Particle-particle interactions in the slurry dictated by factors such as mineralogy, surface chemistry, and $\mathrm{pH}$ govern the inherent settling kinetics, but flocculant addition alters sedimentation rates. Figure 3 shows the settling rates of the tailings after 3 hours, using the above mentioned polymers. 


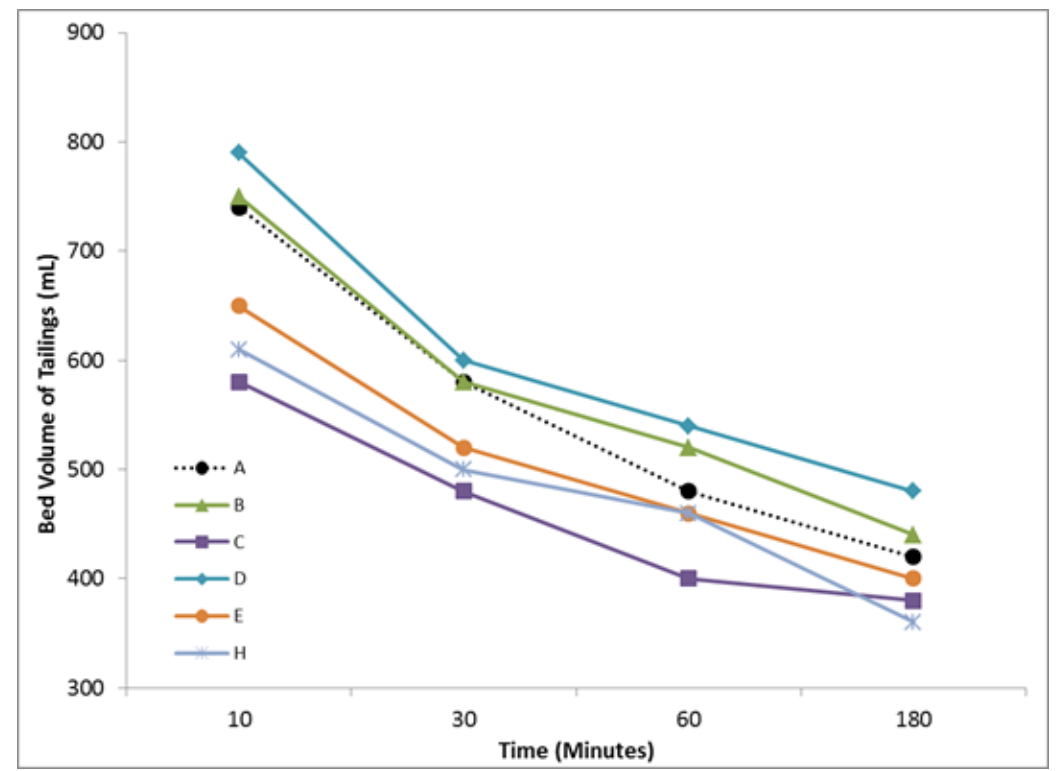

Figure 3 Effect of flocculants on settling rate of processed gold tailings

Although settling starts faster when flocculated with Polymers $C, E$, and $\mathrm{H}$ (bed volume measured at 10 minutes), at the end of 3 hours, Polymers A, B, C, E, and $H$ have bed heights of 420,440, 380, 400 and $380 \mathrm{~mL}$, respectively; the settled bed height has a direct correlation to the underflow solids. The only exception is the cylinder with Polymer $\mathrm{H}$, as the bed height does not contain all the tailings (due to poor flocculation). Figure 4 shows the underflow solids calculated for the tailings treated with select polymers.

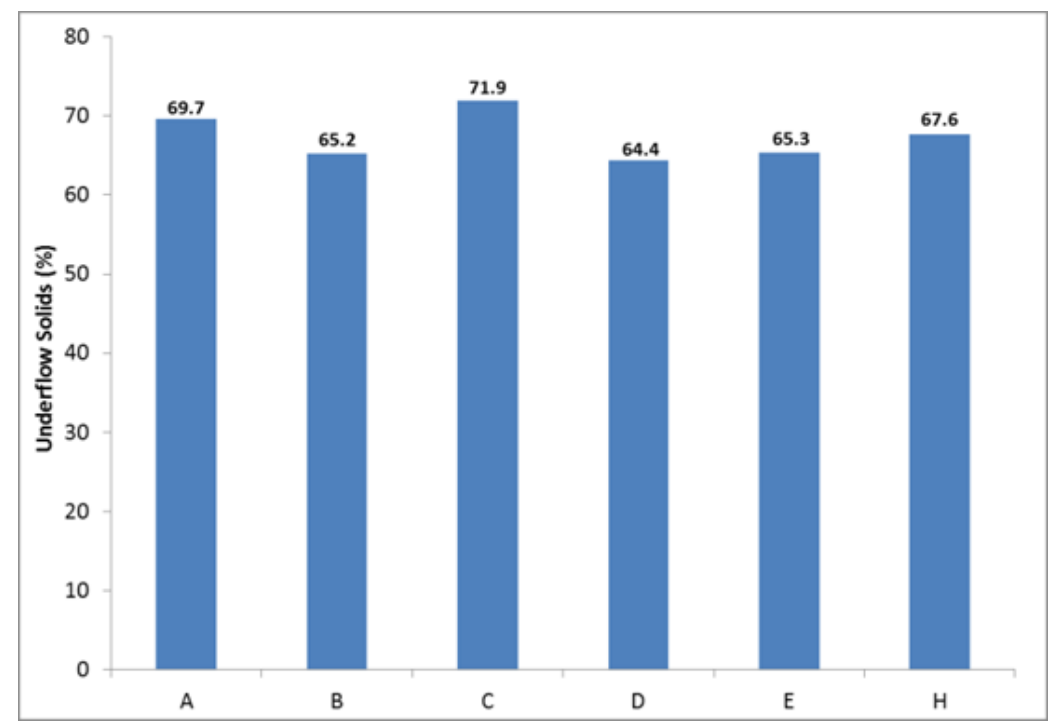

Figure 4 Underflow concentration (\%solids) for processed gold tailings treated with various polymers

\subsection{Dewatering studies}

\subsubsection{Turbidity and suspended solids}

As mentioned previously, increased water recovery has become very important to large numbers of mining operations throughout the world, and it is essential that this recovered water or overflow is clear and free of suspended particles so that it can be easily recycled back into the main stream (Slottee and Biesinger, 2011; Sofra and Boger, 2011). Therefore, it is important that the flocculants not only help in dewatering, but also generate a clear supernatant during the sedimentation/thickening process. Table 1 shows that 
Polymer B has the lowest turbidity value (clarity) of 107 NTU, and very low suspended solids of $0.16 \%$, compared to Polymer A ( $0.44 \%$, incumbent control) or any other polymer.

\subsubsection{Capillary suction time (CST) measurements}

The capillary suction time (CST) test has been used since the 1970 s as a practical yet empirical method for characterising dewatering and the state of colloidal materials in wastewater treatment facilities (Roussel et al., 2006). The main use for the CST is to determine filterability of the flocculated solids after the addition of flocculant aids. CST is the time interval it takes an aqueous solution to traverse between two radial positions in a filter paper (Whatman No. $40,9 \mathrm{~cm}$ ) under the influence of capillary suction. A low CST value implies good sludge dewatering, i.e., the water from the paste releases quickly with little impediment (Roussel et al., 2006). Each measurement was conducted at least in triplicate; the averages are presented in Table 2.

\section{Table 2 Capillary suction time (CST) data for gold tailings treated with various flocculants}

\begin{tabular}{cc}
\hline Sample description & $\begin{array}{c}\text { Capillary suction } \\
\text { time (s) }\end{array}$ \\
\hline Untreated tailings & $56.8 \pm 4.2$ \\
Treated with Polymer A & $69.2 \pm 4.3$ \\
Treated with Polymer B & $6.1 \pm 0.8$ \\
Treated with Polymer C & $23.5 \pm 0.8$ \\
Treated with Polymer D & $5.7 \pm 0.7$ \\
Treated with Polymer E & $7.9 \pm 0.4$ \\
\hline
\end{tabular}

It is observed that Polymers B, D, and E show the lowest CST values when compared to Polymers A or C, signifying that these polymers show better dewatering capabilities. Polymer B shows superior performance, generating the lowest turbidity, suspended solids, and CST values.

\subsection{Stackability}

Stackability of flocculated tailings was primarily measured in two ways: (a) visual observation, and (b) squeeze/compression test.

\subsubsection{Visual observation}

As described in the materials and methods section, after determining the settling kinetics, the supernatant was decanted to its maximum and the settled bed was poured into a tray. Figure 5 shows a representation of the stacking behaviour of the tailings when treated with the selected polymers.

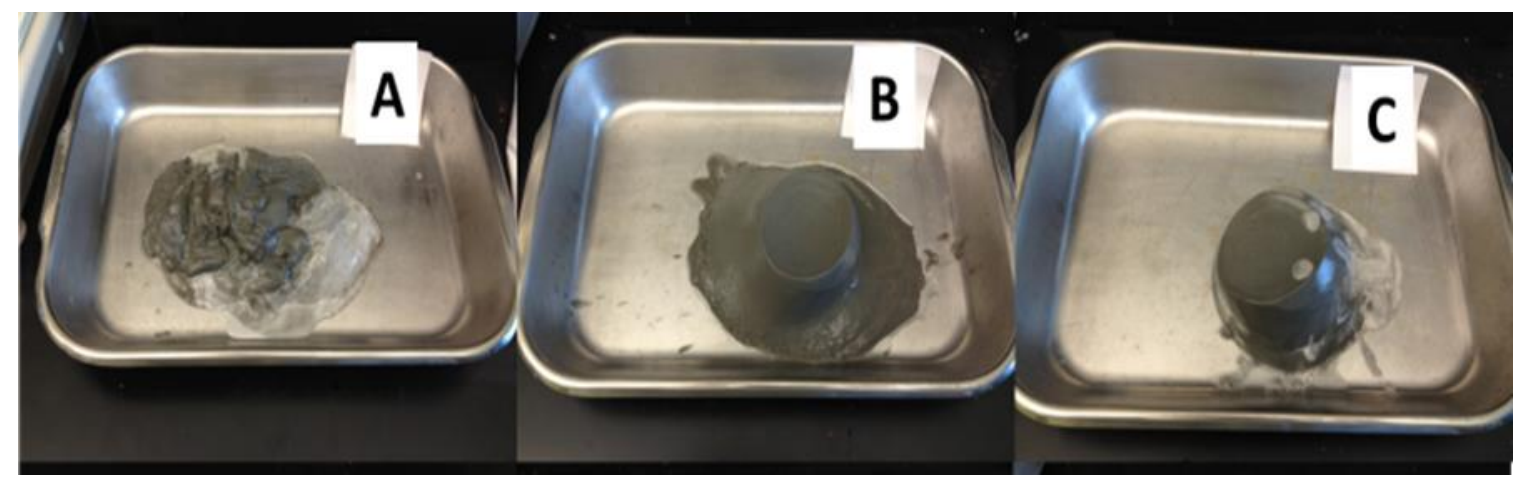

Figure 5 Stacking characteristics of gold tailings flocculated with different polymers 
As seen in Figure 5, Polymer A does not exhibit any stacking characters when compared to Polymers B and C. Polymers $D, E$, and $H$ (not shown) showed stacking behaviour similar to that of Polymer $B$. Polymers $F$ and $\mathrm{G}$ do not show any stacking characteristics due to poor flocculation. Among all examples that exhibit stacking behaviour, Polymer $C$ stacks almost vertically, having no slump at its base, while Polymers $B, D, E$, $\mathrm{H}$, and $\mathrm{J}$ show significant slump at the base. Table 3 shows the slump diameter of tailings flocculated with the tested polymers.

Table 3 Slump diameter of tailings treated with various polymers

\begin{tabular}{cc}
\hline Sample description & $\begin{array}{c}\text { Slump diameter } \\
(\mathbf{c m})\end{array}$ \\
\hline Treated with Polymer A & No stacking \\
Treated with Polymer B & 18 \\
Treated with Polymer C & 11 \\
Treated with Polymer D & 17 \\
Treated with Polymer E & 24 \\
Treated with Polymer H & 21 \\
Treated with Polymer J & 18 \\
\hline
\end{tabular}

\subsubsection{Stackability index using squeeze/compression test}

Squeeze tests are often used in practice as a straightforward technique to determine the flow properties of highly concentrated suspensions such as concrete, molten polymers, ceramic pastes, etc. Most of these materials behave as highly viscous or quasi-plastic fluids, and can be described as Bingham fluids as a first approximation (Scholz, 2005). The squeeze test is a simple compression test. The peak height test deforms a specimen by a preset amount to achieve a deformation of $5 \mathrm{~mm}$, and reports the highest force required to realise that deformation. Table 4 shows the compression test results of the flocculated tailings. For all tests, the solids concentration was between $68-70 \%$.

Table 4 Compression test data for processed gold tailings treated with various polymers

\begin{tabular}{ccc}
\hline Sample description & Force load $(\mathrm{Pa})$ & Remark \\
\hline Untreated tailings & N/A & Paste fails to hold shape \\
Treated with Polymer A & N/A & Paste fails to hold shape \\
Treated with Polymer B & $42.7 \pm 4$ & \\
Treated with Polymer C & $45.8 \pm 4$ & \\
Treated with Polymer D & $40.8 \pm 7$ & \\
Treated with Polymer E & $41.5 \pm 5$ & \\
\hline
\end{tabular}

From the visual tests, it is clear that Polymer A fails to stack the tailings; therefore, it was not possible to collect any data for this and other Polymers like $F$ and $G$. The data suggests that similar stacking ability/strength of tailings is displayed when flocculated using Polymers B, C, D, and E, although Polymer C shows slightly higher force required to deform the stacked tailings. Observations made from the two stackability tests suggest that Polymer $\mathrm{C}$ has a slight advantage (no slump at base) over other Polymers. Although not as good as $C$, Polymer $B$ still shows much greater stacking capabilities compared to Polymers $A, E$, and $H$. 


\subsection{Yield stress, thixotropic recovery, and viscosity from dynamic rheometry}

In the thickening process, as the underflow solids concentration increases, so does the yield stress and the amount of energy the pump must produce to get the paste moving to the disposal area. As the thickened underflow is being transported to the underflow area, its viscosity thins, with part of the reduction in viscosity coming from the flocs being sheared apart. Once the paste reaches the disposal area, the flocs must re-form to allow the slurry to stack effectively (Cross, 1965).

In order to accomplish the goals of high solids, low yield stress, low viscosity, and stackability, the flocculant-particle interactions must be strong enough to withstand the raking arms of the thickener, but still low enough that a pump can overcome the yield stress. Finally, the flocculants must rapidly reform in the absence of shear to avoid slumping upon deposition (Watson et al., 2011; Klein and Pawlik, 2005). Figure 6 shows the yield stress and underflow viscosity (normalised for solids) for the tailings treated with various flocculants.
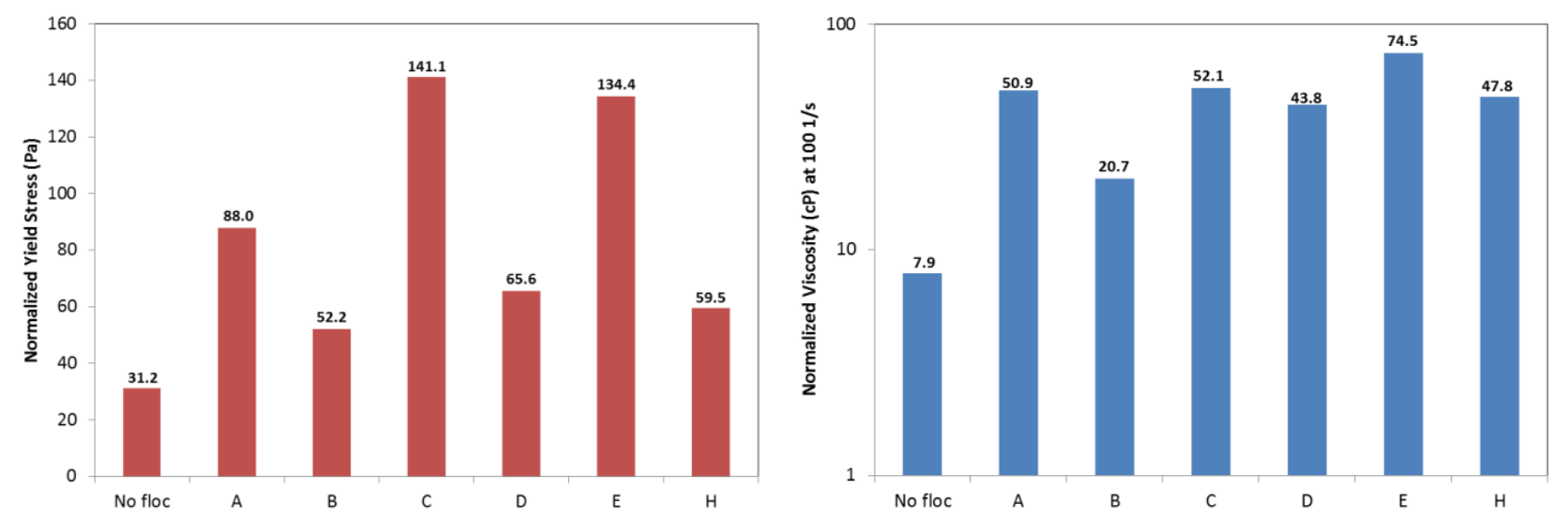

Figure 6 Yield stress data and underflow viscosity at 100 1/s

While all the samples in Figure 6 possess similar underflow solids concentrations, they have very different rheological properties. Underflows resulting from treatments by Polymers A, C, or E, have high yield stresses and viscosities, indicating that they would be more difficult to transport to the disposal area. Meanwhile, Polymers $D$ and, especially, B would be much easier to pump.

Pumpability, however, does not give any information about how well the pastes will stack and dewater. In fact, pumping can damage the floc network, shearing apart the aggregated tailings, and hampering dewaterability. A good flocculant will produce flocs that survive shear and re-form by the time the underflow slurry reaches the disposal area, giving the paste a sufficient yield stress for stacking (Barnes, 1997). The tailings treated with Polymer $B$ had recovered by $8.4 \%$ after 15 minutes and $23.4 \%$ after 30 minutes. Conversely, the tailings treated with Polymer A had only recovered by $0.5 \%$ after 15 minutes and $0.6 \%$ after 30 minutes. Practically, this means that tailings treated with Polymer $B$ would be much more cohesive and stack, while tailings treated with Polymer A would slump and spread instead of stacking.

\subsection{Flocculation study by dynamic light scattering}

Aggregation of tailings was investigated using an inline particle size measurement by dynamic light scattering. Particle size distributions were computed as equivalent-sphere size distributions based on Mie scattering and Fraunhofer diffraction formalisms applied to the scattering data (Farinato et al., 2010). Figures 7 and 8 demonstrate how the particle size distribution (PSD) changes due to the addition of flocculants. 


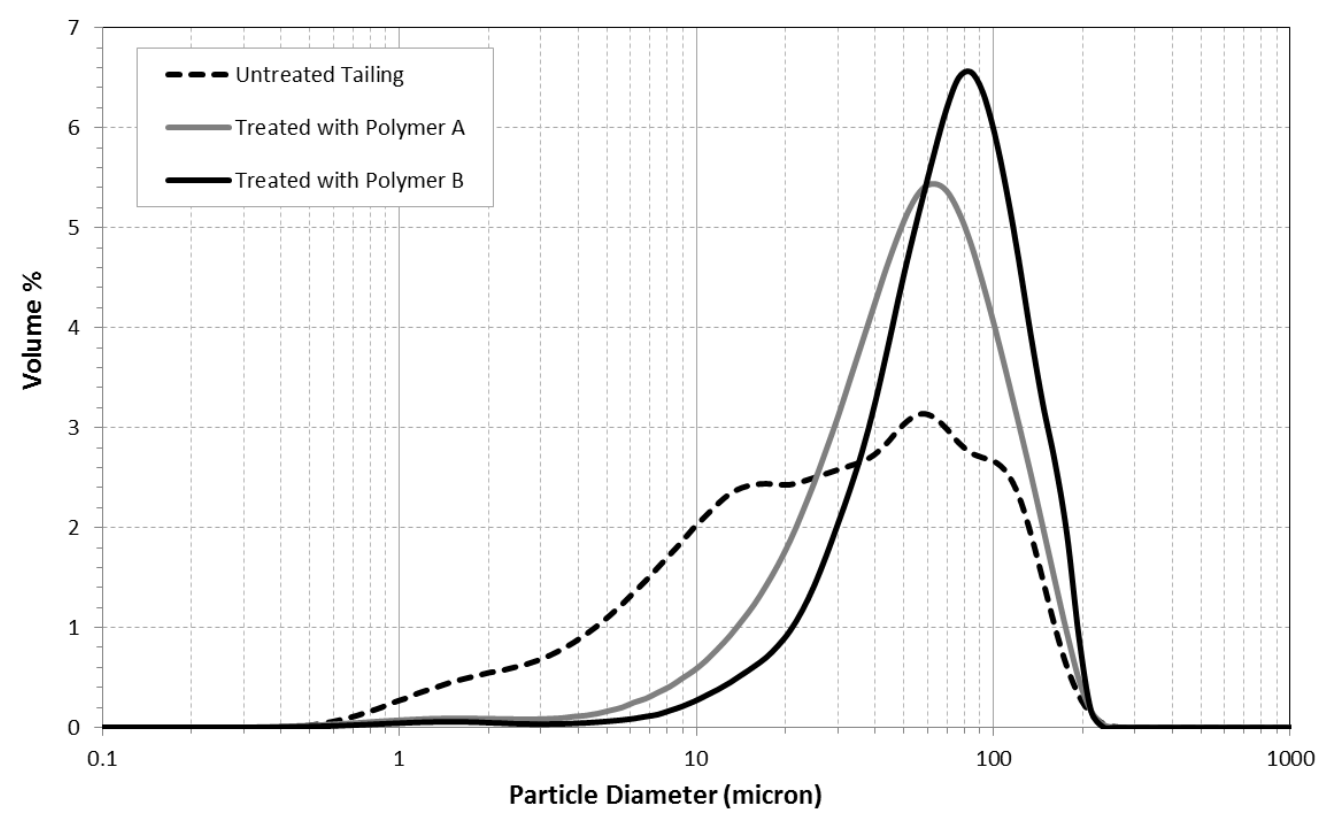

Figure 7 Inline particle size distribution of tailings treated by Polymer A and Polymer B

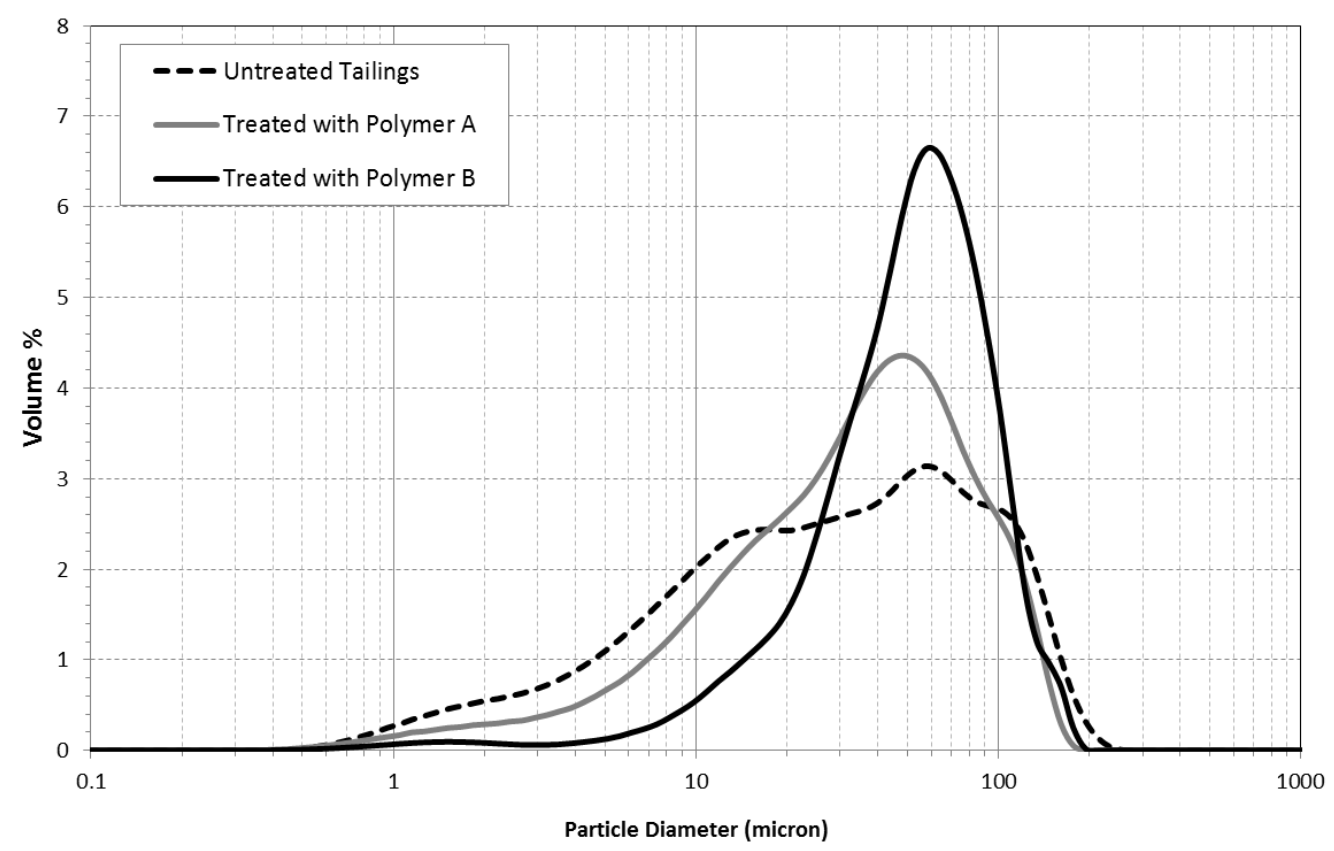

Figure 8 Offline particle size distribution of tailings using Polymer A and Polymer B

A dramatic change in the PSD is seen when flocculants are added. From Figures 7 and 8 , it is observed that Polymer B shows a much narrower PSD compared to Polymer A, signifying a more effective flocculation process. The mean particle size diameter for tailings treated with Polymer $A$ is $\sim 49.6 \mu \mathrm{m}$, while that treated with Polymer B is $\sim 66 \mu \mathrm{m}$. This data implies that Polymer B promotes a higher degree of bridging, which results in the formation of large particle agglomerates. It was also found that Polymer $B$ is more capable capturing ultrafine solids $(<10 \mu \mathrm{m})$ than Polymer $A$, which in turn results in lower solids in the supernatant for tailings treated with Polymer B $(0.44 \%$ for Polymer A versus $0.16 \%$ for Polymer B; see Table 1$)$. Table 5 summarises the mean particle diameter and surface area for some of the flocculated tailings. 
Table 5 PSD data for gold tailings flocculated with various polymers

\begin{tabular}{ccccc}
\hline Polymer & $\begin{array}{c}\text { Mean diameter } \\
(\mu \mathrm{m})\end{array}$ & $\begin{array}{c}\text { PSD, } \mathbf{d}_{10} \\
(\mu \mathrm{m})\end{array}$ & $\begin{array}{c}\text { PSD, } \mathbf{d}_{50} \\
(\mu \mathrm{m})\end{array}$ & $\begin{array}{c}\text { PSD, } \mathbf{d}_{90} \\
(\mu \mathrm{m})\end{array}$ \\
\hline Untreated & 25.64 & 4.6 & 30.2 & 112.8 \\
A & 49.65 & 17.9 & 56.7 & 124.5 \\
B & 66.07 & 28.2 & 74.5 & 143.3 \\
C & 55.63 & 19.2 & 65.1 & 136.6 \\
D & 55.46 & 23.5 & 62.6 & 120.5 \\
E & 46.61 & 17.6 & 54.3 & 107.3 \\
\hline
\end{tabular}

Microscopic studies were also conducted on the flocculated tailings. Figure 9 shows the difference between untreated tailings versus. tailings flocculated with Polymer A and Polymer B.

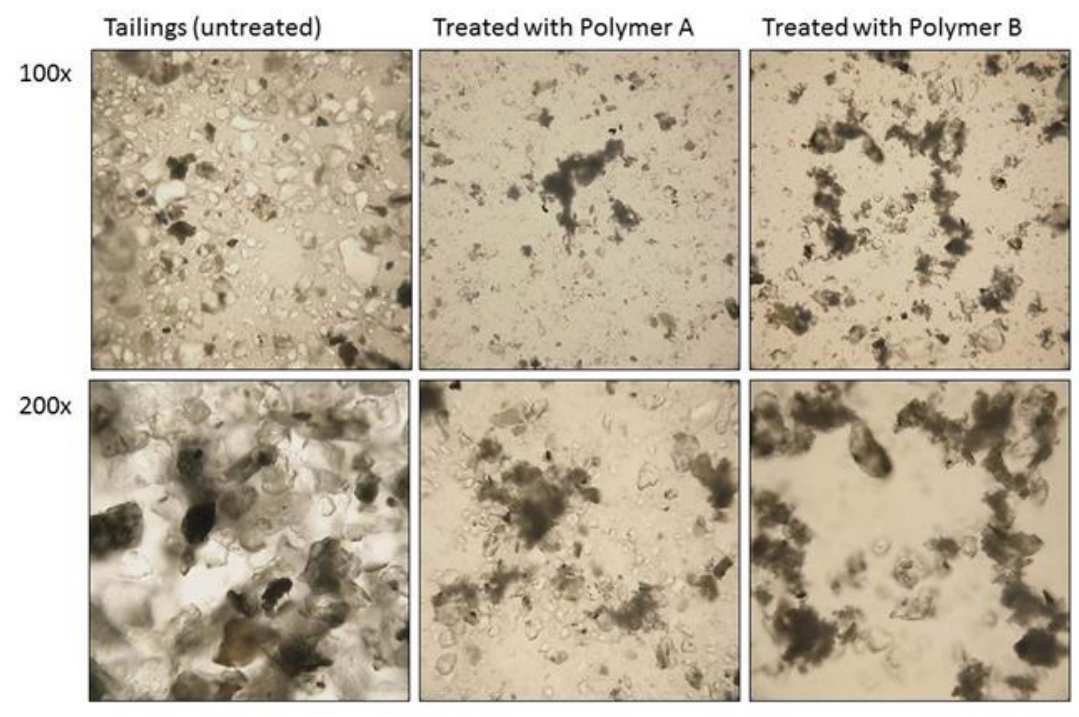

\section{Figure 9 Microscopic studies of flocculated tailings}

From the above, we see that tailings treated with Polymer B show larger agglomerates than those treated with the incumbent Polymer A. This also supports the data obtained from PSD studies.

\section{Conclusions}

In many thickener operations today, flocculant addition has become a common practice in order to achieve desired outcomes with respect to solid-liquid separations. The goal was to develop a flocculant system that can simultaneously target multiple properties of the solid-liquid separation process, such as water recovery (qualitative and quantitative), settling kinetics, and stackability, while still maintaining an acceptable underflow rheology. Table 6 summarises the performance of selected flocculants on key properties of the thickening process. 
Table 6 Summary of characteristics of flocculated tailings

\begin{tabular}{ccccccccc}
\hline & \multicolumn{3}{c}{ Dewatering } & \multicolumn{3}{c}{ Stackability } & \multicolumn{2}{c}{ Rheology } \\
\hline Polymer & $\begin{array}{c}\text { Turbidity } \\
\text { (NTU) }\end{array}$ & $\begin{array}{c}\text { Suspended } \\
\text { solids } \\
(\%)\end{array}$ & $\begin{array}{c}\text { CST } \\
(\mathbf{s})\end{array}$ & $\begin{array}{c}\text { Slump } \\
\text { diameter } \\
(\mathbf{c m})\end{array}$ & $\begin{array}{c}\text { Force } \\
\text { load } \\
(\mathrm{Pa})\end{array}$ & $\begin{array}{c}\text { Mean } \\
\text { diameter } \\
(\mu \mathrm{m})\end{array}$ & $\begin{array}{c}\text { Norm. } \\
\text { yield } \\
\text { stress } \\
(\mathrm{Pa})\end{array}$ & $\begin{array}{c}\text { Norm. } \\
\text { viscosity } \\
\left(\mathbf{c p} @ 100 \mathbf{s}^{-1}\right)\end{array}$ \\
\hline A & $\mathrm{X}$ & 0.44 & 69.2 & $\mathrm{X}$ & $\mathrm{X}$ & 49.65 & 88 & 50.9 \\
B & 107 & 0.16 & 6.1 & 18 & 42.7 & 66.07 & 52.2 & 20.7 \\
C & 897 & 0.24 & 23.5 & 11 & 45.8 & 55.63 & 141.1 & 52.1 \\
D & 163 & 0.09 & 5.7 & 17 & 40.8 & 55.46 & 65.6 & 43.81 \\
E & 364 & 0.13 & 7.9 & 24 & 41.5 & 46.61 & 134.4 & 74.5 \\
\hline
\end{tabular}

Polymer B satisfies all requirements of settling characteristics and stackability and shows superior performance in terms of water recovery, underflow rheology, and thixotropic reformation, and therefore is the flocculant of choice for the tailings used in this study.

Even though the analytical methods used do not yield very precise structural details about the networks that constitute the settled beds, we found that the methods are sensitive enough to identify important differences amongst tailings treated with different flocculants. Treatment of the tailings with the polymers $A$ and $B$ (as well as others) produced substantially different settled beds compared to untreated tailings. Either polymer tended to result in a less dense settled bed; this was more the case with Polymer B than with Polymer A. Dewatering was slightly higher than with untreated tailings for Polymer A while significantly improved for Polymer B (and other polymers). All of these behaviours are consistent with aggregate formation due to polymer bridging of some of the fine solids.

The fact that Polymer B formed less dense settled beds than did Polymer A suggests that those aggregates may be somewhat stronger. However, the configuration of the network formed from tailings treated with Polymer B dewaters measurably better than that made with Polymer A. If the tailings network formed with Polymer A were simply less dense, then one would expect it to dewater more rapidly. Since this was not the case, we have to assume the two networks are not structurally similar.

\section{Acknowledgements}

The authors thank Robert Wilson and Ben Mondesir for their help with obtaining key data, and Kemira for allowing us to publish this study.

\section{References}

American Public Health Association (1999) Standard Methods for the Examination of Water and Wastewater, 20th Edition, American Water Works Association, Water Environment Federation, Washington, D.C.

Barnes, H.A. (1997) Thixotropy - a review, Journal of Non-Newtonian Fluid Mechanics, Vol. 70, pp. 1-33.

Berger, A., Adkins, S., Hess, S., Flanagan, I. and Stocks, P. (2011) Step change improvements in underflow rheology, in Proceedings 14th International Seminar on Paste and Thickened Tailings (Paste2011), R.J. Jewell and A.B. Fourie (eds), 5-7 April 2011, Perth, Australia, Australian Centre for Geomechanics, Perth, pp. 135-141.

Cross, M.M. (1965) Rheology of non-Newtonian fluids: a new flow equation for pseudoplastic systems, Journal of Colloid Science, Vol. 20, pp. 417-437.

Farinato, R., Mahmoudkhani, A., Fenderson, T. and Watson, P. (2010) Segregation and differential settling in flocculated tailings, in Proceedings 2nd International Oil Sands Tailings Conference, 6-8 December, Edmonton, Canada, pp. 58-66.

Hogg, R. (1999) Polymer adsorption and flocculation, Polymers in Mineral Processing, J.S Laskowski (ed), in Proceedings UBC-McGill Bi-Annual International Symposium on Fundamentals of Mineral Processing, Quebec City, Canada, pp. 3-17.

Klein, B. and Pawlik, M. (2005) Rheology modifiers for mineral suspensions, Minerals \& Metallurgical Processing, Vol. 22(2), pp. 83-88.

Malvern Instrument Handbook (1997) Chapter 3 - How the Mastersizer works, MAN 0101, Issue 1.3, pp. 3-1-3-6. 
Mensah-Addai, J. and Ralston, J. (2006) Fundamental and applied dewatering studies of clay mineral dispersions, Interfacial Phenomena in Fine Particle Technology, Z. Xu, Q. Liu, (eds), in Proceedings UBC-McGill-UA International Symposium on Fundamentals of Mineral Processing, Montreal, Canada, pp. 163-177.

Pearse, M.J. (2003) Historical use and future development of chemicals for solid-liquid separation in the mineral processing industry, Minerals Engineering, Vol. 16(2), pp. 103-108.

Pearse, M.J and Barnett, J. (1980) Chemical treatments for thickening and filtration, Filtration+Separation, Vol. 17(5), pp. 460-470.

Roussel, N., Lanos, C. and Toutou, Z. (2006) Identification of Bingham fluid flow parameters using a simple squeeze test, Journal of Non-Newtonian Fluid Mechanics, Vol. 135, pp. 1-7.

Schoenbrunn, F. (2011) Dewatering to higher densities - an industry review, in Proceedings 14th International Seminar on Paste and Thickened Tailings (Paste2011), R.J. Jewell and A.B. Fourie (eds), 5-7 April 2011, Perth, Australia, Australian Centre for Geomechanics, Perth, pp. 19-23.

Scholz, M. (2005) Review of recent trends in capillary suction time (CST) dewaterability testing research, Ind. Eng. Chem. Res, Vol. 44, p. 8157-8167.

Slottee, S. and Biesinger, M.T. (2011) Tailings paste disposal - more than water recovery, SME Annual Meeting 2011, Denver, U.S.A., pp. 1-4.

Sofra, F. and Boger, D.V. (2011) Rheology for thickened tailings and paste - history, state-of-the-art and future directions, in Proceedings 14th International Seminar on Paste and Thickened Tailings (Paste2011), R.J. Jewell and A.B. Fourie (eds), 5-7 April 2011, Perth, Australia, Australian Centre for Geomechanics, Perth, pp. 212-133.

Watson, P., Fenderson, T., Mahmoudkhani, A., Nair, M., Patel, A. and Roberts, G. (2011) Breakage and reformation of flocs in oil sands tailings slurries, in Proceedings Tailings and Mine Waste 2011, Vancouver, Canada, 6-9 November, pp. $293-302$. 
